TRANSACTIONS OF THE

AMERICAN MATHEMATICAL SOCIETY

Volume 356, Number 7, Pages 2871-2887

S 0002-9947(03)03363-4

Article electronically published on November 12, 2003

\title{
THE ABC THEOREM FOR HIGHER-DIMENSIONAL FUNCTION FIELDS
}

\author{
LIANG-CHUNG HSIA AND JULIE TZU-YUEH WANG
}

\begin{abstract}
We generalize the $\mathrm{ABC}$ theorems to the function field of a variety over an algebraically closed field of arbitrary characteristic which is nonsingular in codimension one. We also obtain an upper bound for the minimal order sequence of Wronskians over such function fields of positive characteristic.
\end{abstract}

\section{INTRODUCTION}

Let $k$ be an algebraically closed field of characteristic $p \geq 0$. Let $x_{0}, x_{1}, x_{2} \in k[t]$ be non-zero relatively prime polynomials such that $x_{0}+x_{1}=x_{2}$. If $x_{0} / x_{1}$ is not a constant for the case where $p=0$, or $x_{0} / x_{1}$ is not a $p$-th power in $k(t)$ for the case where $p>0$, then Mason's "ABC theorem" $\mathrm{Ma}$ asserts that

$$
\max \operatorname{deg}\left\{x_{0}, x_{1}, x_{2}\right\} \leq N_{1}\left(x_{0} x_{1} x_{2}\right)-1 \text {. }
$$

We denote by $N_{1}(f)$ the number of distinct zeroes of $f$ for any non-zero polynomial $f \in k[t]$. In fact, Mason's theorem is proved for any function field of transcendence degree one over $k$. With more variables involved in the sum of functions $x_{0}+\cdots+x_{n}=x_{n+1}, n \geq 2$, Mason's theorem was generalized by Voloch [Vol, Brownawell-Masser [BM], and the second author [Wa1]. In the case of higher-dimensional function fields, Shapiro-Sparer SS proved an ABC theorem for $\mathbb{C}\left[X_{1}, \ldots, X_{N}\right]$. Using techniques from Nevanlinna theory, Noguchi $[\mathrm{No}$ was able to generalize the $\mathrm{ABC}$ theorem for the function field of a smooth variety over $\mathbb{C}$. It is well known from Vojta's dictionary $\mathrm{Voj}$ that the $\mathrm{ABC}$ theorem corresponds to the truncated second main theorem in Nevanlinna theory. A Nevanlinna second main theorem for affine algebraic manifolds defined over $\mathbb{C}$ was obtained by Stoll-Wong [SW] and Ye [Ye].

The ABC theorem has many applications in the study of Diophantine geometry. For example, it was used to study the $S$-integral points on projective spaces minus hyperplanes. It also has applications in obtaining finiteness properties of integral or rational points on curves or varieties defined over function fields. It is also important to establish some sort of $\mathrm{ABC}$ theorem for a more general algebraic varieties. For example, Buium $[\mathrm{Bu}]$ obtained an $\mathrm{ABC}$ theorem for any affine open subset of an abelian variety with trace zero. We refer to $[\mathrm{Bu}]$ for a more general definition of $\mathrm{ABC}$ theorems, and to Wa3 for the relations between $\mathrm{ABC}$ theorem and integral points on projective spaces minus hyperplanes.

Received by the editors April 15, 2003.

2000 Mathematics Subject Classification. Primary 11J97; Secondary 11J61.

Key words and phrases. ABC theorem, function fields, Diophantine approximation.

(C)2003 American Mathematical Society 
The major goal of this paper is to generalize the ABC theorem over a higherdimensional function field of positive characteristic. In fact, our method treats both the cases of zero characteristic as well as positive characteristic. Let $V$ be a projective variety over an algebraically closed field $k$ of characteristic $p \geq 0$ and which is non-singular in codimension one. Let $K=k(V)$ be its function field which is separably generated over $k$. Let $N$ be the dimension of $V$ over $k$. Let $M_{K}$ denote the set of prime divisors (irreducible subvrieties of codimension one) of $V$ over $k$. We state our main result (Theorem 1 ) in the case of positive charactersitic as follows.

Theorem. Let $L_{1}, \ldots, L_{q}, q \geq n+1$, be linear forms in $n+1$ variables over $k$ which are in general position. Let $\mathfrak{X}=\left[x_{0}, \ldots, x_{n}\right] \in \mathbb{P}^{n}(K)$ be such that $x_{0}, \ldots, x_{n}$ are linearly independent over $K^{p^{m}}$ for some $m \in \mathbb{N}$. Then, for any fixed finite subset $S$ of $M_{K}$, the following inequality holds:

$$
\begin{aligned}
& (q-n-1) h\left(x_{0}, \ldots, x_{n}\right) \\
& \leq \sum_{i=1}^{q} \sum_{\mathfrak{p} \notin S} \operatorname{deg} \mathfrak{p} \min \left\{n p^{m-1}, \operatorname{ord}_{\mathfrak{p}}\left(L_{i}(\mathfrak{X})\right)-\min _{0 \leq j \leq n}\left\{\operatorname{ord}_{\mathfrak{p}}\left(x_{j}\right)\right\}\right\} \\
& \quad+\frac{n(n+1)}{2} p^{m-1}\left(\operatorname{deg} \mathcal{K}_{V}+O(N) \operatorname{deg} V+\sum_{\mathfrak{p} \in S} \operatorname{deg} \mathfrak{p}\right),
\end{aligned}
$$

where $O(N)=0$ if $N=1$ and $O(N)=N$ if $N>1$.

For the definition of the height $h\left(x_{0}, \ldots, x_{n}\right)$ of $\mathfrak{X} \in \mathbb{P}^{n}(K)$ and the canonical divisor $\mathcal{K}_{V}$, see Section 4.

Our approach uses algebraic methods which are close to the methods used in GV], Wa1 and Wa2]. One of the key ingredients is the study of a generalized Wronskian in positive characteristic which was introduced by H. Hasse and F. K. Schmidt [HS in the curve case and by Okugama Ok in the case of multivariables. In $\mathrm{GV}]$ Garcia and Voloch gave a criterion for the linear independence of elements of $K$ over $K^{p^{m}}$ in terms of the non-vanishing property of a generalized Wronskian. We will generalize their criterion to the multi-variable case and follow the ideas in Wa1, Wa2 to refine their criterion. We will also give a formula of changing variables for a generalized Wronskian. This formula is necessary for estimating the vanishing order of the generalized Wronskian along any prime divisor in $V$. Similar results were obtained in Wa1, [Wa2] in the curve case. Since more variables are involved, more efforts are needed in deducing the results. These will be treated in Section 2 and Section 3.

The major difficulty in proving the higher-dimensional ABC theorem is that the ramification theory is more complicated in the higher-dimensional case than in the curve case. In our case, $V$ is regarded as a finite ramified cover over the $N$-dimensional projective space $\mathbb{P}^{N}$. The ramified divisors of the cover $V \rightarrow \mathbb{P}^{N}$ will arise naturally when estimating the vanishing order of a generalized Wronskian. We will briefly review in Section 4 the theory of ramification which will be used in our situation.

In Section 5, we deduce our main result. The major task is to estimate the vanishing order of a generalized Wronskian along an arbitrary prime divisor of $V$. We are able to bound the vanishing order in terms of the degree of the ramification 
divisor of $V$. Once this is done, we then apply our results in Sections 2 and 3 to prove our main theorem.

In the final section, we give a refinement of the orders of the iterated derivative (Theorem 2) appearing in the generalized Wronskian. For the definition of the iterated derivatives, see Section 2. In the course of the proof of our main result, these orders will affect the sharpness of the error term in the ABC theorem. We believe that these refinements should be useful in improving the error term in some circumstances.

Throughout this paper, we fix $k$ to be an algebraically closed field of characteristic $p \geq 0$. We let $V$ denote a projective variety of dimension $N$ over $k$ which is nonsingular in codimension one. Let $K=k(V)$ be its function field.

\section{WRONSKIANS IN POSITIVE CHARACTERISTIC}

In this section we will generalize results in GV], Wa1, Wa2 on Wronskians over 1-dimensional function fields to the higher-dimensional case. The methods used here are similar to those three papers.

Let $\boldsymbol{z}=\left\{z_{1}, \ldots, z_{N}\right\}$ be a set of transcendental basis of $K$ such that $K$ is a finite separable extension over $k\left(z_{1}, \ldots, z_{N}\right)$. Let $\alpha_{j}, \beta_{j}$ be positive integers. The iterated Hasse partial derivatives (iterated derivatives for short) $D_{z_{j}}^{\alpha_{j}}$ are defined by the formula $D_{z_{j}}^{\alpha_{j}}\left(z_{j}^{\beta_{j}}\right)=\left(\begin{array}{c}\beta_{j} \\ \alpha_{j}\end{array}\right) z_{j}^{\beta_{j}-\alpha_{j}}$, and $D_{z_{j}}^{\alpha_{j}}\left(z_{m}^{\beta_{m}}\right)=0$ if $j \neq m$. Then the iterated derivatives are defined on $k\left(z_{1}, \ldots, z_{N}\right)$ and they can be uniquely extended to $K$ as $K$ is separable over $k\left(z_{1}, \ldots, z_{N}\right)$. We will use the same notations $D_{z_{j}}^{\alpha_{j}}$ to denote their extensions.

Throughout the paper, we will use the bold Greek letters $\boldsymbol{\alpha}, \boldsymbol{\beta}, \ldots, \boldsymbol{\epsilon}$, etc. to denote integer vectors whose entries are non-negative integers. For $\boldsymbol{\alpha}=\left(\alpha_{1}, \ldots, \alpha_{N}\right)$ and $\boldsymbol{\beta}=\left(\beta_{1}, \ldots, \beta_{N}\right)$, we use the convention $\boldsymbol{\alpha} \leq \boldsymbol{\beta}(\boldsymbol{\alpha}<\boldsymbol{\beta})$ to mean that $\alpha_{i} \leq \beta_{i}$ (resp., $\alpha_{i}<\beta_{i}$ ) for all $i=1, \ldots, N$. Set $D_{z}^{\boldsymbol{\alpha}}:=D_{z_{1}}^{\alpha_{1}} \ldots D_{z_{N}}^{\alpha_{N}}$; then the order of $D_{\boldsymbol{z}}^{\boldsymbol{\alpha}}$ is defined to be $|\boldsymbol{\alpha}|:=\sum_{j=1}^{N} \alpha_{j}$. The iterated derivatives satisfy the following elementary properties. (See $[\mathrm{GV}], \mathrm{Ok}$.)

Proposition 2.1. (a) $D_{\boldsymbol{z}}^{\boldsymbol{\alpha}}(x y)=\sum_{\boldsymbol{\beta}+\boldsymbol{\gamma}=\boldsymbol{\alpha}} D_{\boldsymbol{z}}^{\boldsymbol{\beta}}(x) D_{\boldsymbol{z}}^{\boldsymbol{\gamma}}(y)$, for all $x, y \in K$.

(b) $D_{\boldsymbol{z}}^{\boldsymbol{\alpha}} D_{\boldsymbol{z}}^{\boldsymbol{\beta}}(x)=\left(\begin{array}{c}\alpha_{1}+\beta_{1} \\ \beta 1\end{array}\right) \ldots\left(\begin{array}{c}\alpha_{N}+\beta_{N} \\ \beta_{N}\end{array}\right) D_{\boldsymbol{z}}^{\boldsymbol{\alpha}+\boldsymbol{\beta}}(x)$, for all $x \in K$.

(c) Let $\boldsymbol{t}=\left\{t_{1}, \ldots, t_{N}\right\}$ be another separable transcendental basis of $K$ over $k$. Then we have

$$
D_{z_{j}}(x)=\sum_{i=1}^{N} \frac{\partial t_{i}}{\partial z_{j}} D_{t_{i}}(x)
$$

for all $x \in K$.

For the rest of this section we assume that $k$ is of positive characteristic $p$. Set

$$
\begin{aligned}
& K_{m}=\left\{x \in K \mid D_{z_{j}}^{\alpha_{j}}(x)=0 \text { for } 1 \leq \alpha_{j}<p^{m}, 1 \leq j \leq N\right\}, \quad \text { and } \\
& K_{\infty}=\left\{x \in K \mid D_{\boldsymbol{z}}^{\alpha}(x)=0 \text { for all }|\boldsymbol{\alpha}| \geq 1\right\} .
\end{aligned}
$$

Proposition 2.2. $\quad$ (a) For all $m \geq 1, K_{m}$ is a field. Moreover, $D_{z_{j}}^{p^{m}}, 1 \leq$ $j \leq N$, are partial derivations on $K_{m}$ and $K_{m+1}=\left\{x \in K_{m} \mid D_{z_{j}}^{p^{m}}(x)=\right.$ 0$, for all $1 \leq j \leq N\}$.

(b) $K_{m}=K^{p^{m}}$ and $K_{\infty}=k$. 
Proof. (a) follows from [GV], Proposition 1, and (b) follows from [HS], Satz 10, and induction on the transcendence degree of $K$ over $k$.

Proposition 2.3. The field extension degree $\left[K: K_{m}\right]$ is $p^{N m}$. Furthermore, if $\left\{z_{1}, \ldots, z_{N}\right\}$ is a set of transcendental basis of $K$ over $k$, then $K$ is a separable extension of $K_{m}$ and the set $\left\{z_{1}^{\epsilon_{1}} \ldots z_{N}^{\epsilon_{N}} \mid 0 \leq \epsilon_{i} \leq p^{m}-1,1 \leq i \leq N\right\}$ forms a basis of $K$ over $K_{m}$.

Proof. To ease the notation, we denote $k\left(z_{1}, \ldots, z_{N}\right)$ by $F$. Note that $F_{m}=$ $k\left(z_{1}^{p^{m}}, \ldots, z_{N}^{p^{m}}\right)$ since $k$ is perfect. Note that the map $\phi_{m}=\left(x \mapsto x^{p^{m}}\right)$ induces a field isomorphism from $K$ to $K_{m}$. Also, $F$ is isomorphic to $F_{m}$ via this isomorphism. Since $K$ is separable over $F$, it follows easily that $K_{m}$ is separable over $F_{m}$ and $\left[K_{m}: F_{m}\right]=[K: F]$. The separability of $K_{m}$ over $F_{m}$ implies that $K_{m}$ is linearly disjoint from $F$ over $F_{m}$ (see, for example, [La1, Chap. X, §6]). By the linear disjointness, we have $\left[K_{m} F: F\right]=\left[K_{m}: F_{m}\right]=[K: F]$. Hence, $K=K_{m} F$. It's clear that $\left\{z_{1}^{\epsilon_{1}} \ldots z_{N}^{\epsilon_{N}} \mid 0 \leq \epsilon_{i} \leq p^{m}-1,1 \leq i \leq N\right\}$ is a basis for $F$ over $F_{m}$. Now our claim follows by the linear disjointness between $K_{m}$ and $F$ over $F_{m}$.

We now formulate a linear independence criterion for a given set of elements of $K$ over $K_{m}$.

Lemma 1. Let $x_{0}, \ldots, x_{n}$ be elements of $K$. Then $x_{0}, \ldots, x_{n}$ are linearly independent over $K_{m}$ if and only if there exists a sequence of integer vectors $\boldsymbol{\epsilon}^{i} \leq\left(p^{m}-1, \ldots\right.$, $\left.p^{m}-1\right)$ with $0=\left|\boldsymbol{\epsilon}^{0}\right| \leq\left|\boldsymbol{\epsilon}^{1}\right| \cdots \leq\left|\boldsymbol{\epsilon}^{n}\right|$ such that

$$
\operatorname{det}\left(D_{\boldsymbol{z}}^{\epsilon^{i}}\left(x_{j}\right)\right)_{0 \leq i, j \leq n} \neq 0 \text {. }
$$

Remark. Lemma 1 can be proved by induction as in [GV] for the proof of the case $N=1$. Here we present a different proof by using Proposition 2.3.

Proof. By Proposition 2.3 the set of elements $\left\{\boldsymbol{z}^{\boldsymbol{\epsilon}} \mid 0 \leq \boldsymbol{\epsilon} \leq\left(p^{m}-1, \ldots, p^{m}-1\right)\right\}$ is a basis of $K$ over $K_{m}$. For this chosen basis, we note that $D_{\boldsymbol{z}}^{\boldsymbol{\epsilon}} \boldsymbol{z}^{\boldsymbol{\epsilon}}=1$ and $D_{\boldsymbol{z}}^{\boldsymbol{\alpha}} \boldsymbol{z}^{\boldsymbol{\epsilon}}=0$ if $\alpha_{i}>\epsilon_{i}$ for some $1 \leq i \leq N$. We fix an order among the integer vectors $\epsilon$ 's and set $\boldsymbol{B}=\left(D_{\boldsymbol{z}}^{\boldsymbol{\alpha}} \boldsymbol{z}^{\boldsymbol{\epsilon}}\right)$ to be the square matrix with entry $D_{\boldsymbol{z}}^{\boldsymbol{\alpha}} \boldsymbol{z}^{\boldsymbol{\epsilon}}$ where $0 \leq \boldsymbol{\alpha}, \boldsymbol{\epsilon} \leq$ $\left(p^{m}-1, \ldots, p^{m}-1\right)$. Then, a direct computation shows that $\boldsymbol{B}$ is an invertible matrix. For any given elements $x_{0}, \ldots, x_{n}$ of $K$, there exists an $(n+1) \times p^{N m}$-matrix $\boldsymbol{M}$ over $K_{m}$ with $\boldsymbol{M}=\left(m_{i \epsilon}\right), 0 \leq i \leq n, 0 \leq \boldsymbol{\epsilon} \leq\left(p^{m}-1, \ldots, p^{m}-1\right)$, such that

$$
x_{i}=\sum_{\epsilon} m_{i \epsilon} z^{\epsilon}, \quad i=0, \ldots, n,
$$

where the sum is over all integer vectors $\boldsymbol{\epsilon}$ such that $0 \leq \boldsymbol{\epsilon} \leq\left(p^{m}-1, \ldots, p^{m}-1\right)$. By Proposition 2.1(a) and Proposition 2.2, we see that

$$
D_{\boldsymbol{z}}^{\boldsymbol{\alpha}} x_{i}=\sum_{\boldsymbol{\epsilon}} m_{i \boldsymbol{\epsilon}} D_{\boldsymbol{z}}^{\boldsymbol{\alpha}} \boldsymbol{z}^{\boldsymbol{\epsilon}} \quad \text { for } i=0, \ldots, n \text { and all } 0 \leq \boldsymbol{\alpha} \leq\left(p^{m}-1, \ldots, p^{m}-1\right) .
$$

Hence, $\left(D_{\boldsymbol{z}}^{\boldsymbol{\alpha}} x_{i}\right)=\boldsymbol{B} \boldsymbol{M}^{t}$. Now it is clear that $x_{0}, \ldots, x_{n}$ are linear independent over $K_{m}$ if and only if $\boldsymbol{M}$ has rank equal to $n+1$, and this is equivalent to the rank of the matrix $\left(D_{\boldsymbol{z}}^{\boldsymbol{\alpha}} x_{i}\right)$ being $n+1$. Thus there exists a sequence of integer vectors $\boldsymbol{\epsilon}^{0}, \boldsymbol{\epsilon}^{1}, \ldots, \boldsymbol{\epsilon}^{n}$ with $0 \leq \boldsymbol{\epsilon}^{i} \leq\left(p^{m}-1, \ldots, p^{m}-1\right)$ such that the determinant of the matrix $\left(D_{z}^{\epsilon^{i}} x_{j}\right)$ is not identically zero. We may rearrange the indices so that $0=\left|\boldsymbol{\epsilon}^{0}\right| \leq\left|\boldsymbol{\epsilon}^{1}\right| \leq \cdots \leq\left|\boldsymbol{\epsilon}^{n}\right|$. This completes the proof of Lemma 1 . 
Lemma 2. If $x_{0}, \ldots, x_{n} \in K$ and are linearly independent over $K_{m}$, then there exists a sequence of integer vectors $\boldsymbol{\epsilon}^{i}$ with $0=\left|\boldsymbol{\epsilon}^{0}\right| \leq\left|\boldsymbol{\epsilon}^{1}\right| \cdots \leq\left|\boldsymbol{\epsilon}^{n}\right|$ and $\left|\boldsymbol{\epsilon}^{i}\right| \leq$ ip ${ }^{m-1}$ such that

$$
\operatorname{det}\left(D_{\boldsymbol{z}}^{\epsilon^{i}}\left(x_{j}\right)\right)_{0 \leq i, j \leq n} \neq 0
$$

Remark 1. The bound is sharp in some cases. For example, let $K=k\left(z_{1}, \ldots, z_{N}\right)$ and the sequence of functions be $1, z_{1}^{p^{m-1}} f_{1}, \ldots, z_{1}^{(p-1) p^{m-1}} f_{p-1}$, where $f_{i} \in K^{p^{m}}$ and $f_{i} \neq 0$. Then the minimal $\boldsymbol{\epsilon}^{i}$ satisfying Lemma 2 is $\boldsymbol{\epsilon}^{i}=\left(i p^{m-1}, 0, \ldots, 0\right)$.

Remark 2. If $K$ is of characteristic zero, then $\left|\boldsymbol{\epsilon}^{i}\right| \leq i$. A proof can be found in Fu].

Proof. The proof will be done by induction on $n$. When $n=0$, this is trivial. Let $n$ be a positive integer. Let $x_{0}, \ldots, x_{n} \in K$ be linearly independent over $K_{m}$ and assume that the lemma holds for $x_{0}, \ldots, x_{n-1}$. That is, there exist integer vectors $\boldsymbol{\epsilon}^{0}, \ldots, \boldsymbol{\epsilon}^{n-1}$ with $\left|\boldsymbol{\epsilon}^{i}\right| \leq i p^{m-1}$ such that $\operatorname{det}\left(D_{\boldsymbol{z}}^{\boldsymbol{\epsilon}^{i}}\left(x_{j}\right)\right)$ is not identically zero. Let the integer vectors $\boldsymbol{\epsilon}^{i}$ be fixed for $i=0, \ldots, n-1$. If the lemma does not hold for $x_{0}, \ldots, x_{n}$, then the following $n+1$ vectors

$$
\underline{\boldsymbol{x}}_{i}=\left(x_{i}, D_{\boldsymbol{z}}^{\boldsymbol{\epsilon}^{1}}\left(x_{i}\right), \ldots, D_{\boldsymbol{z}}^{\boldsymbol{\epsilon}^{(n-1)}}\left(x_{i}\right), \ldots, D_{\boldsymbol{z}}^{\boldsymbol{\alpha}}\left(x_{i}\right), \ldots\right), \quad \text { with }|\boldsymbol{\alpha}| \leq n p^{m-1}
$$

are linearly dependent over $K$. Thus, there exist $a_{0}, \ldots, a_{n} \in K$ such that

$$
\sum_{i=0}^{n} a_{i} D_{\boldsymbol{z}}^{\boldsymbol{\alpha}}\left(x_{i}\right)=0 \quad \text { for }|\boldsymbol{\alpha}| \leq n p^{m-1} .
$$

Since $x_{0}, \ldots, x_{n-1}$ are linearly independent over $K_{m}$, by the induction hypothesis, the first $n$ vectors $\underline{\boldsymbol{x}}_{0}, \ldots, \underline{\boldsymbol{x}}_{n-1}$ are linearly independent over $K$. Hence $a_{n} \neq 0$, and without loss of generality, we may assume that $a_{n}=1$. We shall show that $a_{i} \in K_{m}$, and then $(2.1)$ with $\boldsymbol{\alpha}=(0, \ldots, 0)$ will give a contradiction to the assumption that $x_{0}, \ldots, x_{n}$ are linearly independent over $K_{m}$.

To show that $a_{i} \in K_{m}$, it suffices to show that $D_{z_{j}}^{r}\left(a_{i}\right)=0$ for $1 \leq r \leq p^{m-1}$ and $1 \leq j \leq N$. This will be done by induction on $r$. For $r=1$, applying the operator $D_{z_{j}}$ to (2.1), then by Proposition 2.1 and (2.1) we have

$$
\sum_{i=0}^{n-1}\left(D_{z_{j}} a_{i}\right) D_{\boldsymbol{z}}^{\boldsymbol{\alpha}}\left(x_{i}\right)=0, \quad \text { for }|\boldsymbol{\alpha}| \leq n p^{m-1}-1 .
$$

In particular,

$$
\sum_{i=0}^{n-1}\left(D_{z_{j}} a_{i}\right) D_{\boldsymbol{z}}^{\boldsymbol{\alpha}}\left(x_{i}\right)=0, \quad \text { for }|\boldsymbol{\alpha}| \leq(n-1) p^{m-1} .
$$

By the induction hypothesis that the $n$ vectors $\underline{\boldsymbol{x}}_{0}, \ldots, \underline{\boldsymbol{x}}_{n-1}$ are linearly independent over $K$, we conclude that $D_{z_{j}} a_{i}=0$ for $j=1, \ldots, N$ and $i=0, \ldots, n-1$.

Assume that for $i=0, \ldots, n-1$

$$
D_{z_{j}}^{r}\left(a_{i}\right)=0 \text { for } 1 \leq r<b \leq p^{m-1} .
$$

Now applying $D_{z_{j}}^{b}$ to $(2.1)$, we deduce that

$$
\sum_{i=0}^{n-1}\left(D_{z_{j}}^{b} a_{i}\right) D_{\boldsymbol{z}}^{\boldsymbol{\alpha}}\left(x_{i}\right)=0, \quad \text { for }|\boldsymbol{\alpha}| \leq(n-1) p^{m-1}
$$


by Proposition 2.1, (2.1) and (2.2). Consequently, the linear independency of $\underline{\boldsymbol{x}}_{0}, \ldots, \underline{\boldsymbol{x}}_{n-1}$ over $K$ implies that $D_{z_{j}}^{b} a_{i}=0$ for $1 \leq b \leq p^{m-1}, j=1, \ldots, N$, and $i=0, \ldots, n-1$. This completes the induction steps and proves the lemma.

\section{Further Properties of Wronskians}

We retain the notation used in Section 2. In order to simplify the notation, we let the boldface letter $\boldsymbol{f}$ denote the vector $\left(f_{0}, \ldots, f_{n}\right)$ of $K^{n+1}$. For any iterated derivatives $D^{\boldsymbol{\alpha}}$, we set $D^{\boldsymbol{\alpha}} \boldsymbol{f}=\left(D^{\boldsymbol{\alpha}} f_{0}, \ldots, D^{\boldsymbol{\alpha}} f_{n}\right)$ and more generally, for $D=$ $\sum_{\boldsymbol{\alpha}} a_{\boldsymbol{\alpha}} D^{\boldsymbol{\alpha}}$, we set $D \boldsymbol{f}=\sum_{\boldsymbol{\alpha}} a_{\boldsymbol{\alpha}} D^{\boldsymbol{\alpha}} \boldsymbol{f}$, where $a_{\boldsymbol{\alpha}} \in K$ and the sum is a finite sum. We now denote $k K^{p^{m}}$ to be $k$ if $p=0$ or $K^{p^{m}}$ if $p>0$. By results of Section 2 , if $f_{0}, \ldots, f_{n}$ are linearly independent over $k K^{p^{m}}$, then there exists a sequence of integer vectors $\epsilon^{0}, \ldots, \epsilon^{n}$ with $0=\left|\epsilon^{0}\right|<\left|\epsilon^{1}\right| \leq \cdots \leq\left|\epsilon^{n}\right|$ such that the Wronskian

$$
\operatorname{det}\left(D_{\boldsymbol{z}}^{\boldsymbol{\epsilon}^{i}} f_{j}\right)_{0 \leq i, j \leq n}
$$

does not vanish identically. We choose a sequence of integer vectors $\epsilon^{i}$ so that they are minimal in the following sense: $\boldsymbol{\epsilon}^{0}=(0, \ldots, 0)$; if $\boldsymbol{\epsilon}^{0}, \ldots, \boldsymbol{\epsilon}^{i-1}$ are chosen, then we choose $\boldsymbol{\epsilon}^{i}$ with $\left|\boldsymbol{\epsilon}^{i}\right|$ minimal such that the row vectors $D_{\boldsymbol{z}}^{\epsilon^{0}} \boldsymbol{f}, \ldots, D_{\boldsymbol{z}}^{\epsilon^{i}} \boldsymbol{f}$ are linearly independent over $K$. Therefore, if $\boldsymbol{\beta}^{0}, \ldots, \boldsymbol{\beta}^{n}$ are integer vectors with $\left|\boldsymbol{\beta}^{0}\right| \leq \cdots \leq$ $\left|\boldsymbol{\beta}^{n}\right|$ such that the vectors $D_{\boldsymbol{z}}^{\boldsymbol{\beta}^{0}} \boldsymbol{f}, \ldots, D_{\boldsymbol{z}}^{\boldsymbol{\beta}^{n}} \boldsymbol{f}$ are linearly independent over $K$, then $\left|\boldsymbol{\epsilon}^{i}\right| \leq\left|\boldsymbol{\beta}^{i}\right|$ for each $i=0, \ldots, n$. For a fixed $\boldsymbol{f}=\left(f_{0}, \ldots, f_{n}\right) \in K^{n+1}$, the symbols $\epsilon^{0}, \ldots, \epsilon^{n}$ are reserved for any chosen sequence of minimal integer vectors in the above sense with respect to the set of transcendental basis $\boldsymbol{z}=\left\{z_{1}, \ldots, z_{N}\right\}$. In our discussion below, the $K$-vector $\boldsymbol{f}$ (or $\mathfrak{X}$ ) is fixed, hence we will call $\left\{\boldsymbol{\epsilon}^{0}, \ldots, \boldsymbol{\epsilon}^{n}\right\}$ a sequence of minimal integer vectors (with respect to $\boldsymbol{z}$ ). Let $\boldsymbol{t}=\left\{t_{1}, \ldots, t_{N}\right\}$ be another separable transcendental basis for $K$ over $k$. Let $\boldsymbol{\alpha}$ be a given integer vector. For integer vector $\boldsymbol{\beta}$ with $|\boldsymbol{\beta}|=|\boldsymbol{\alpha}|$ we define $\prod_{\boldsymbol{\alpha}}^{\boldsymbol{\beta}} \frac{\partial \boldsymbol{t}}{\partial \boldsymbol{z}}$ to be the coefficient of $D_{t}^{\beta} \boldsymbol{f}$ in the following formula:

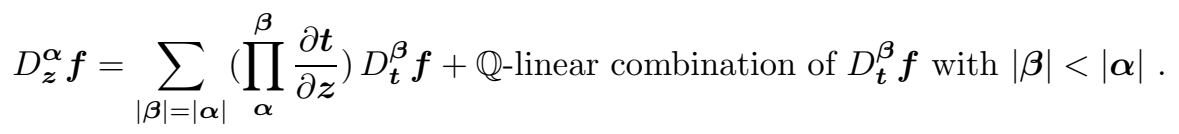

Then $\prod_{\boldsymbol{\alpha}}^{\boldsymbol{\beta}} \frac{\partial \boldsymbol{t}}{\partial \boldsymbol{z}}$ is a $\mathbb{Q}$-linear combination of $\prod_{i=1}^{|\boldsymbol{\alpha}|} \frac{\partial t_{a(i)}}{\partial z_{b(i)}}$, where $a(i)$ and $b(i)$ are some integers between 1 and $|\boldsymbol{\alpha}|$. For example, if $\boldsymbol{\alpha}=(2,1,0, \ldots, 0)$ and $\boldsymbol{\beta}=(1,2,0, \ldots, 0)$, then $\prod_{\alpha}^{\boldsymbol{\beta}} \frac{\partial t}{\partial \boldsymbol{z}}=2 \frac{\partial t_{1}}{\partial z_{1}} \frac{\partial t_{2}}{\partial z_{1}} \frac{\partial t_{2}}{\partial z_{2}}+\frac{\partial t_{1}}{\partial z_{2}}\left(\frac{\partial t_{2}}{\partial z_{1}}\right)^{2}$.

Note that a sequence of minimal integer vectors is not unique. However the sequence of their orders $\left|\boldsymbol{\epsilon}^{0}\right|, \ldots,\left|\boldsymbol{\epsilon}^{n}\right|$ is unique with respect to $\boldsymbol{z}$. In fact, we will show that the sequence of orders $\left|\boldsymbol{\epsilon}^{0}\right|, \ldots,\left|\boldsymbol{\epsilon}^{n}\right|$ is independent of the choice of separable transcendental basis.

Proposition 3.1. $\quad$ (a) If $g_{i}=\sum a_{i j} f_{j}$ with $\left(a_{i j}\right) \in G L_{n+1}(k)$, then

$$
\operatorname{det}\left(D_{\boldsymbol{z}}^{\epsilon^{i}} g_{j}\right)=\operatorname{det}\left(a_{i j}\right) \operatorname{det}\left(D_{\boldsymbol{z}}^{\epsilon^{i}} f_{j}\right)
$$

(b) If $h \in K$, then

$$
\operatorname{det}\left(D_{\boldsymbol{z}}^{\epsilon^{i}} h f_{j}\right)=h^{n+1} \operatorname{det}\left(D_{\boldsymbol{z}}^{\epsilon^{i}} f_{j}\right)
$$

(c) The sequence of minimal order $\left\{\left|\boldsymbol{\epsilon}^{0}\right|,\left|\boldsymbol{\epsilon}^{1}\right|, \ldots,\left|\boldsymbol{\epsilon}^{n}\right|\right\}$ is independent of the choice of a separable transcendental basis for $K$ over $k$. Furthermore, if 
$\boldsymbol{t}=\left\{t_{1}, \ldots, t_{N}\right\}$ is another transcendental basis, then the following formula for the determinant of the Wronskian holds:

$$
\operatorname{det}\left(D_{\boldsymbol{z}}^{\epsilon^{i}} f_{j}\right)=\operatorname{det}\left(\sum_{|\boldsymbol{\alpha}|=\left|\boldsymbol{\epsilon}^{i}\right|}\left(\prod_{\boldsymbol{\epsilon}^{i}}^{\boldsymbol{\alpha}} \frac{\partial \boldsymbol{t}}{\partial \boldsymbol{z}}\right) D_{\boldsymbol{t}}^{\boldsymbol{\alpha}} f_{j}\right) .
$$

Before we give a proof of Proposition 3.1, we make the following remark.

Remark. Let $\mathcal{E}_{d}$ denote the subspace generated by the set of vectors $\left\{D_{\boldsymbol{z}}^{\boldsymbol{\alpha}} \boldsymbol{f}|| \boldsymbol{\alpha} \mid \leq\right.$ $d\}$ over $K$ and let $\lambda_{d}=\operatorname{dim}_{K} \mathcal{E}_{d}$.

By definition, it is clear that $1=\lambda_{0} \leq \lambda_{1} \leq \cdots \leq \lambda_{i} \leq \cdots$. Since $f_{0}, \ldots, f_{n}$ are linearly independent over $K_{m}$, it follows by Lemma 1 that there exists a positive integer $d_{s}$ such that $\lambda_{d_{s}}=n+1$. Let $d_{1}, d_{2}, \ldots, d_{s}$ be the indices such that $1=$ $\lambda_{0}=\cdots<\lambda_{d_{1}}=\cdots<\lambda_{d_{2}}=\cdots$, etc., and $\lambda_{d_{s}}=n+1$. By the definition of the sequence of minimal integer vectors, we see that the sequence of integers $0<d_{1}<\cdots<d_{s}$ are the orders of minimal integer vectors. Moreover, the set of $K$-vectors $\left\{\boldsymbol{f}, D_{\boldsymbol{z}}^{\boldsymbol{\epsilon}^{1}} \boldsymbol{f}, \ldots, D_{\boldsymbol{z}}^{\boldsymbol{\epsilon}^{\lambda_{i}}} \boldsymbol{f}\right\}$ forms a basis of $\mathcal{E}_{d_{i}}$ for $1 \leq i \leq s$.

Proof. (a) This is a standard property of Wronskians.

(b) Note that the $(i+1)$-th row of the Wronskian $\left(D_{\boldsymbol{z}}^{\epsilon^{i}} h f_{j}\right)$ is the row vector $D_{\boldsymbol{z}}^{\boldsymbol{\epsilon}^{i}} h \boldsymbol{f}$. We claim that each row of the Wronskian can be replaced by the row vector $h D_{z}^{\epsilon^{i}} f$ for $0 \leq i \leq n$ without changing the determinant of the Wronskian. For $i=0, D_{\boldsymbol{z}}^{\epsilon^{0}} h \boldsymbol{f}=h \boldsymbol{f}$ and the claim holds automatically. Assume that the claim holds for all rows $D_{\boldsymbol{z}}^{\epsilon^{i}} h \boldsymbol{f}$ such that $\left|\boldsymbol{\epsilon}^{i}\right|<d_{j}$ for some $j$ with $0<j \leq s$. Assume that $\left|\boldsymbol{\epsilon}^{r}\right|=d_{j}$, then by Proposition 2.1(a),

$$
D_{\boldsymbol{z}}^{\boldsymbol{\epsilon}^{r}} h \boldsymbol{f}=h D_{\boldsymbol{z}}^{\boldsymbol{\epsilon}^{r}} \boldsymbol{f}+\sum_{|\boldsymbol{\beta}|<\left|\epsilon^{r}\right|}\left(D_{\boldsymbol{z}}^{\boldsymbol{\alpha}} h\right)\left(D_{\boldsymbol{z}}^{\boldsymbol{\beta}} \boldsymbol{f}\right) .
$$

The set of vectors $\left\{D_{\boldsymbol{z}}^{\boldsymbol{\beta}} \boldsymbol{f}|| \boldsymbol{\beta}|<| \boldsymbol{\epsilon}^{r} \mid=d_{j}\right\}$ is contained in $\mathcal{E}_{d_{j-1}}$. They are in the subspace generated by $D_{\boldsymbol{z}}^{\epsilon^{l}} \boldsymbol{f}$ with $\left|\boldsymbol{\epsilon}^{l}\right|<d_{j}$. Hence, replacing the row vector $D_{\boldsymbol{z}}^{\epsilon^{r}} h \boldsymbol{f}$ by $h D_{\boldsymbol{z}}^{\epsilon^{r}} \boldsymbol{f}$ does not change the determinant of the Wronskian. The claim now follows. It is clear that assertion (b) follows from the claim.

(c) By Proposition 2.1, we have the following identity:

$$
D_{z}^{\boldsymbol{\alpha}} \boldsymbol{f}=\sum_{|\boldsymbol{\beta}|=|\boldsymbol{\alpha}|}\left(\prod_{\boldsymbol{\alpha}}^{\boldsymbol{\beta}} \frac{\partial \boldsymbol{t}}{\partial \boldsymbol{z}}\right) D_{\boldsymbol{t}}^{\boldsymbol{\beta}} \boldsymbol{f}+\text { linear combination of } D_{\boldsymbol{t}}^{\boldsymbol{\beta}} \boldsymbol{f} \text { with }|\boldsymbol{\beta}|<|\boldsymbol{\alpha}| .
$$

We also have

$$
D_{\boldsymbol{t}}^{\gamma} \boldsymbol{f}=\sum_{|\boldsymbol{\delta}|=|\gamma|}\left(\prod_{\gamma}^{\boldsymbol{\delta}} \frac{\partial \boldsymbol{z}}{\partial \boldsymbol{t}}\right) D_{\boldsymbol{z}}^{\boldsymbol{\delta}} f+\text { linear combination of } D_{\boldsymbol{z}}^{\boldsymbol{\delta}} \boldsymbol{f} \text { with }|\boldsymbol{\delta}|<|\gamma| .
$$

Let $\mathcal{F}_{d}$ denote the subspace generated by the set of vectors $\left\{D_{t}^{\boldsymbol{\alpha}} \boldsymbol{f}|| \boldsymbol{\alpha} \mid \leq d\right\}$ over $K$. It is not hard to see from (3.1) and (3.2) that $\mathcal{E}_{d} \subseteq \mathcal{F}_{d}$ as well as $\mathcal{F}_{d} \subseteq \mathcal{E}_{d}$. Thus, the orders of minimal integer vectors $0=d_{0}<d_{1}<\cdots<d_{s}$ are invariant under the change of coordinates. This proves the first part of (c).

The second part of (c) is also a consequence of (3.1) and (3.2). Note that the first row of the Wronskian $\left(D_{\boldsymbol{z}}^{\boldsymbol{\epsilon}^{i}} f_{j}\right)$ is the row vector $\boldsymbol{f}$ which remains the same after 
the change of coordinates. Suppose that we have replaced the row vectors $D_{\boldsymbol{z}}^{\boldsymbol{\epsilon}^{i} \boldsymbol{f}}$ by the following sum:

$$
\sum_{|\boldsymbol{\alpha}|=\left|\boldsymbol{\epsilon}^{i}\right|}\left(\prod_{\boldsymbol{\epsilon}^{i}}^{\boldsymbol{\alpha}} \frac{\partial \boldsymbol{t}}{\partial \boldsymbol{z}}\right) D_{\boldsymbol{t}}^{\boldsymbol{\alpha}} \boldsymbol{f} \quad \text { for all } \boldsymbol{\epsilon}^{i} \text { with }\left|\boldsymbol{\epsilon}^{i}\right|<d_{j}
$$

without changing the determinant of the Wronskian . Let $\left|\boldsymbol{\epsilon}^{r}\right|=d_{j}$, then by (3.1) we have

$$
D_{\boldsymbol{z}}^{\boldsymbol{\epsilon}^{r}} \boldsymbol{f}=\sum_{|\boldsymbol{\alpha}|=\left|\boldsymbol{\epsilon}^{r}\right|}\left(\prod_{\boldsymbol{\epsilon}^{r}}^{\boldsymbol{\alpha}} \frac{\partial \boldsymbol{t}}{\partial \boldsymbol{z}}\right) D_{\boldsymbol{t}}^{\boldsymbol{\alpha}} \boldsymbol{f}+A_{l},
$$

where $A_{l}$ is a linear combination of $D_{\boldsymbol{t}}^{\boldsymbol{\alpha}} \boldsymbol{f}$ with $|\boldsymbol{\alpha}|<d_{j}$ and hence they are in $\mathcal{F}_{d_{j-1}}$. Therefore, replacing $D_{\boldsymbol{z}}^{\boldsymbol{\epsilon}^{r}} \boldsymbol{f}$ by the sum $\sum_{|\boldsymbol{\alpha}|=\left|\boldsymbol{\epsilon}^{r}\right|}\left(\prod_{\boldsymbol{\epsilon}^{r}}^{\boldsymbol{\alpha}} \frac{\partial \boldsymbol{t}}{\partial \boldsymbol{z}}\right) D_{\boldsymbol{t}}^{\boldsymbol{\alpha}} \boldsymbol{f}$ does not change the determinant. Moreover, the row vectors $\left\{\sum_{|\boldsymbol{\alpha}|=\left|\boldsymbol{\epsilon}^{i}\right|}\left(\prod_{\boldsymbol{\epsilon}^{i}}^{\boldsymbol{\alpha}} \frac{\partial \boldsymbol{t}}{\partial \boldsymbol{z}}\right) D_{\boldsymbol{t}}^{\boldsymbol{\alpha}} \boldsymbol{f}|| \boldsymbol{\epsilon}^{i} \mid \leq d_{j}\right\}$ must be linearly independent over $K$ and generate $\mathcal{F}_{d_{j}}$, otherwise, the determinant would be zero which is not the case. Inductively, we can replace each row of the Wronskian by the sum as above and the second part of (c) follows.

Proposition 3.2. Let $\mathfrak{p}$ be an irreducible subvariety of $V$ of codimension one. Let $P$ be a smooth point of $\mathfrak{p}$ and let $\boldsymbol{t}=\left\{t_{1}, \ldots, t_{N}\right\}$ be a local coordinate system of $P$ in an open neighborhood $U$. If $f$ is a non-constant element of $K$ and regular along $\mathfrak{p}$, then for any integer vector $\boldsymbol{\alpha} \geq 0$,

$$
\operatorname{ord}_{\mathfrak{p}} \frac{D_{\boldsymbol{t}}^{\boldsymbol{\alpha}}(f)}{f} \geq-\min \left\{|\boldsymbol{\alpha}|, \operatorname{ord}_{\mathfrak{p}} f\right\} .
$$

Proof. Let $\mathcal{O}_{V, P}$ denote the local ring at $P$. Over some smaller neighborhood of $P$ contained in $U, \mathfrak{p}$ has a local defining equation $g \in \mathcal{O}_{V, P}$ and $f=u g^{\operatorname{ord}_{\mathfrak{p}} f}$ where $u \in \mathcal{O}_{V, P}$ and $u$ does not vanish along $\mathfrak{p}$. By Proposition 2.1,

$$
D_{\boldsymbol{t}}^{\boldsymbol{\alpha}}\left(u g^{\operatorname{ord}_{\mathfrak{p} f}}\right)=\sum_{\boldsymbol{\alpha}=\boldsymbol{\beta}+\boldsymbol{\gamma}} D_{\boldsymbol{t}}^{\boldsymbol{\beta}} u D_{\boldsymbol{t}}^{\boldsymbol{\gamma}} g^{\operatorname{ord}_{\mathfrak{p}} f} .
$$

The following inequality is clear:

$$
\operatorname{ord}_{\mathfrak{p}} D_{\boldsymbol{t}}^{\gamma} g^{\operatorname{ord}_{\mathfrak{p}} f} \geq \max \left\{0, \operatorname{ord}_{\mathfrak{p}}(f)-|\gamma|\right\} .
$$

Hence

$$
\operatorname{ord}_{\mathfrak{p}} \frac{D_{\boldsymbol{t}}^{\boldsymbol{\alpha}}(f)}{f} \geq-\min \left\{|\gamma|, \operatorname{ord}_{\mathfrak{p}} f\right\} \geq-\min \left\{|\boldsymbol{\alpha}|, \operatorname{ord}_{\mathfrak{p}} f\right\}
$$

\section{Height over a FUnCtion Field}

For the rest of the paper, we shall fix a projective embedding of $V$ such that $V \subset \mathbb{P}^{M}$ for some positive integer $M$. Let $M_{K}$ denote the set of prime divisors (irreducible subvarieties of codimension one) of $V$. In this section, we recall the definition of the Weil height of points of $\mathbb{P}^{n}(K)$ as well as some basic facts from algebraic geometry.

Let $\mathfrak{p} \in M_{K}$ be a prime divisor. As $V$ is non-singular in codimension one, the local ring $\mathcal{O}_{\mathfrak{p}}$ at $\mathfrak{p}$ is a discrete valuation ring. For each $x \in K^{*}$ its order $\operatorname{ord}_{\mathfrak{p}} x$ at $\mathfrak{p}$ is well defined. We can associate to $x$ its divisor

$$
(x)=\sum_{\mathfrak{p} \in M_{K}} \underset{\mathfrak{p}}{\operatorname{ord}}(x) \mathfrak{p}=(x)_{0}-(x)_{\infty},
$$


where $(x)_{0}$ is the zero divisor of $x$ and $(x)_{\infty}$ is the polar divisor of $x$, respectively. Let $\operatorname{deg} \mathfrak{p}$ denote the projective degree of $\mathfrak{p}$ in $\mathbb{P}^{M}$. Then the sum formula

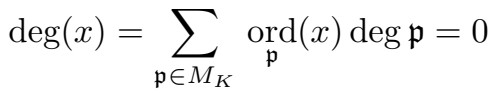

holds for all $x \in K^{*}$.

Given $\mathfrak{X}=\left[x_{0}, \ldots, x_{n}\right] \in \mathbb{P}^{n}(K)$ with $x_{0}, \ldots, x_{n} \in K$ not all zero, the (logarithmic) height of $\mathfrak{X}$ is defined by the following formula:

$$
h(\mathfrak{X})=-\sum_{\mathfrak{p}} \min _{i}\left\{\underset{\mathfrak{p}}{\operatorname{ord}}\left(x_{i}\right)\right\} \operatorname{deg} \mathfrak{p} .
$$

Note that $\mathfrak{X}$ defines a rational map $\mathfrak{X}: V \cdots \rightarrow \mathbb{P}^{n}$ over $k$. The height of $\mathfrak{X}$ can also be interpreted as the projective degree of $\mathfrak{X}^{-1}(H)$, where $H$ is any hyperplane of $\mathbb{P}^{n}$ which does not contain the image of $\mathfrak{X}$ (for a proof, see [La2, Chap. 3, Prop. 3.2 ] or [Se, Chap. 1, 2.2]).

In what follows, we shall fix an $(M-N-1)$-plane $\mathbb{P}^{M-N-1} \subset \mathbb{P}^{M}$ which is disjoint from $V$. Let $\mathbb{P}^{N}$ be an $N$-plane which is complementary to $\mathbb{P}^{M-N-1}$ and let $\pi: V \rightarrow \mathbb{P}^{N}$ be the projection from $\mathbb{P}^{M-N-1}$. We may choose $\pi$ so that it is a finite, separable morphism over $k$. Then the degree of $\pi$ is equal to the projective degree of $V$ in $\mathbb{P}^{M}$. Let $V^{0}$ denote the smooth locus of $V$. Let $\pi^{0}$ denote the restriction of $\pi$ on $V^{0}$. Because $V$ is non-singular in codimension one, the group

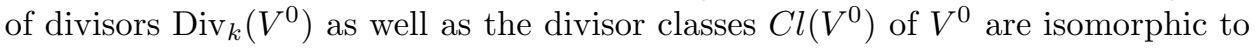
$\operatorname{Div}_{k}(V)$ and $C l(V)$, respectively.

Let $\mathcal{K}_{V^{0}}$ denote the canonical divisor class of $V^{0}$ and let $\mathcal{R}_{\pi}^{0}$ denote the divisor class containing the branch divisor of $\pi^{0}$. Then, the identity

$$
\mathcal{K}_{V^{0}}=\mathcal{R}_{\pi}^{0}+\left(\pi^{0}\right)^{*}\left(\mathcal{K}_{\mathbb{P}^{N}}\right)
$$

holds as usual, where $\mathcal{K}_{\mathbb{P}^{N}}$ denotes the canonical divisor class of $\mathbb{P}^{N}$. Let $H^{0}$ denote a hyperplane section of $V^{0}$. By abuse of notation, we will also denote $H^{0}$ as the divisor class containing $H^{0}$. Then the above identity can by expressed in terms of $H^{0}$ as follows:

$$
\mathcal{K}_{V^{0}}=\mathcal{R}_{\pi}^{0}-(N+1) H^{0}
$$

We also have the corresponding identity on $V$ :

$$
\mathcal{K}_{V}=\mathcal{R}_{\pi}-(N+1) H
$$

where $\mathcal{K}_{V}, \mathcal{R}_{\pi}$ and $H$ denote the divisor class obtained by taking the Zariski closure of each codimension one irreducible subvariety in the representing divisors of $\mathcal{K}_{V^{0}}, \mathcal{R}_{\pi}^{0}$ and $H^{0}$ in $V$, respectively. In the following, we will also call $\mathcal{R}_{\pi}$ the branch divisor of $\pi$. Note that $H$ is also a hyperplane section of $V$.

\section{The truncated SeCond Main theorem}

We retain the notation from Section 4 . Let $\left[Z_{0}, \ldots, Z_{N}\right]$ be a set of homogeneous coordinates of $\mathbb{P}^{N}$. Without loss of generality, we may assume that the coordinate hyperplane $H_{0}$ of $\mathbb{P}^{n}$, defined by equation $Z_{0}=0$, is not contained in $\pi\left(\mathcal{R}_{\pi}\right)$. Let $z_{i}=\frac{Z_{i}}{Z_{0}}$ for $1 \leq i \leq N$. Then the set of functions $\pi^{*}\left(z_{i}\right)=z_{i} \circ \pi, 1 \leq i \leq n$, forms a set of transcendental basis of $K$. By abuse of the notation, we will still let $z_{i}$ denote $\pi^{*}\left(z_{i}\right)$ in $K$. Then, $K$ is finite and separable over $k\left(z_{1}, \ldots, z_{N}\right)$ with degree equal to the degree of $\pi$.

Definition. Let $Q=\sum_{\mathfrak{p}} m_{\mathfrak{p}} \mathfrak{p}$ be a divisor of $V$. We define $\operatorname{deg} Q=\sum_{\mathfrak{p}} m_{\mathfrak{p}} \operatorname{deg} \mathfrak{p}$. 
Theorem 1. Let $\mathfrak{X}=\left[x_{0}, \ldots, x_{n}\right] \in \mathbb{P}^{n}(K)$. Let $q \geq n+1$ and let $L_{1}, \ldots, L_{q}$ be linear forms in $n+1$ variables over $k$ which are in general position, that is, any $n+1$ elements of $L_{1}, \ldots, L_{q}$ are linearly independent over $k$. Let $S$ be any fixed finite subset of $M_{K}$. Suppose that $x_{0}, \ldots, x_{n}$ are linearly independent over $k K^{p^{m}}$, then

$$
\begin{aligned}
& (q-n-1) h\left(x_{0}, \ldots, x_{n}\right) \\
& \leq \sum_{i=1}^{q} \sum_{\mathfrak{p} \notin S} \operatorname{deg} \mathfrak{p} \min \left\{n c(m, k), \operatorname{ord}_{\mathfrak{p}}\left(L_{i}(\mathfrak{X})\right)-\min _{0 \leq j \leq n}\left\{\operatorname{ord}_{\mathfrak{p}}\left(x_{j}\right)\right\}\right\} \\
& \quad+\frac{n(n+1)}{2} c(m, k)\left(\operatorname{deg} \mathcal{K}_{V}+O(N) \operatorname{deg} V+\sum_{\mathfrak{p} \in S} \operatorname{deg} \mathfrak{p}\right),
\end{aligned}
$$

where the constant $c(m, k)=1$ if $p=0$ and $c(m, k)=p^{m-1}$ otherwise; and the constant $O(N)=0$ if $N=1$ and $O(N)=N$ if $N>1$.

Remark 1 . If $k$ is of positive characteristic, then $c(m, k)$ can be formulated in terms of the minimal order $\left|\epsilon^{i}\right|$ which has a better bound as described in Theorem 2 in Section 6 .

Remark 2. If $V$ is a smooth curve over $k(N=1)$, then $\operatorname{deg} \mathcal{K}_{V}=2 g(V)-2$ where $g(V)$ denotes the genus of $V$.

Remark 3. When $k=\mathbb{C}$, Theorem 1 is similar to the result of Noguchi [No]. However, the error term in Theorem $1, \frac{n(n+1)}{2}\left(\operatorname{deg} \mathcal{K}_{V}+O(N) \operatorname{deg} V+\sum_{\mathfrak{p} \in S} \operatorname{deg} \mathfrak{p}\right)$, is more explicit.

As a special case of Theorem 1, we have the following result for polynomial rings.

Corollary. Let $K=k\left(z_{1}, \ldots, z_{N}\right)$ and let $f_{1}, \ldots, f_{n} \in k\left[z_{1}, \ldots, z_{N}\right]$ which are relatively prime. Let $L_{1}, \ldots, L_{q}$ be linear forms in $n+1$ variables over $k$ and in general position. If $f_{0}, \ldots, f_{n}$ are linearly independent over $k K^{p^{m}}$, then

$$
\begin{aligned}
& (q-n-1) \max \left\{\operatorname{deg} f_{0}, \ldots, \operatorname{deg} f_{n}\right\} \\
& \leq \sum_{i=1}^{q} \sum_{P} \min \left\{n c(m, k), \operatorname{ord}_{P}\left(L_{i}\left(f_{0}, \ldots, f_{n}\right)\right)\right\} \operatorname{deg} P,
\end{aligned}
$$

where $P$ runs through all irreducible polynomials in $k\left[z_{1}, \ldots, z_{N}\right]$.

Remark 4. Shapiro and Sparer [SS] dealt with the special case that $k=\mathbb{C}$ under the assumption that $f_{0}, \ldots, f_{n}$ are pairwise coprime.

Proof of Theorem 1. Let $U_{0}=\mathbb{P}^{N} \backslash H_{0}$. Fix any prime divisor $\mathfrak{p}$ of $V$. Since $V$ is non-singular in codimension one by assumption, the intersection $\mathfrak{p}^{0}=\mathfrak{p} \cap V^{0}$ is non-empty. We will choose any point $w$ which is in the smooth locus of $\mathfrak{p}$ and choose a neighborhood $U_{w, \mathfrak{p}}$ of $w$ such that $\mathfrak{p}$ is defined by the zero locus of a single function $g_{\mathfrak{p}}$. Let $\boldsymbol{t}=\left\{t_{1}, \ldots, t_{N}\right\}$ be a local coordinate system of $w$ in $U_{w, \mathfrak{p}}$. The point $w$ and the local coordinate system $\boldsymbol{t}$ can be chosen to satisfy one of the following conditions:

(i) If $\mathfrak{p}$ is contained in the support of the branched divisor $\mathcal{R}_{\pi}$, then $\pi(\mathfrak{p}) \neq H_{0}$ by assumption. Let $w \in \mathfrak{p}^{0} \cap \pi^{-1}\left(U_{0}\right)$ and $U_{w, \mathfrak{p}} \subset \pi^{-1}\left(U_{0}\right)$. We may choose $t_{1}=g_{\mathfrak{p}}$ and the branch locus $\pi(\mathfrak{p})$ is defined by $a t_{1}^{e_{\mathfrak{p}}}$ near $\pi(w)$ for some $a \in K$ which has no pole or zero along $\mathfrak{p}$. Then $\left\{a t_{1}^{e_{\mathfrak{p}}}, t_{2}, \ldots, t_{N}\right\}$ forms a local coordinate system in an open neighborhood of $\pi(w)$. (See [BPV], p. 41, for a proof which also works in 
finite characteristic.) Write $u=a t_{1}^{e_{\mathfrak{p}}}$, then $\frac{\partial u}{\partial t_{1}} \neq 0$ with $\operatorname{ord}_{\mathfrak{p}}\left(\frac{\partial u}{\partial t_{1}}\right) \geq e_{\mathfrak{p}}-1$ since $\pi$ is separable.

(ii) If $\mathfrak{p}$ is not branched and $\pi(\mathfrak{p}) \neq H_{0}$, then we may choose any $w \in \mathfrak{p}^{0} \cap$ $\pi^{-1}\left(U_{0}\right)$. There exists an open neighborhood $U_{w, \mathfrak{p}} \subset \pi^{-1}\left(U_{0}\right)$ of $w$ such that $\left\{t_{1}=z_{1}, \ldots, t_{N}=z_{N}\right\}$ forms a local coordinate system of $U_{w, \mathfrak{p}}$.

(iii) If $\mathfrak{p}$ is not branched but $\pi(\mathfrak{p})=H_{0}$, then we may choose a $w$ and an open neighborhood $U_{w, \mathfrak{p}}$ of $w$ such that $\left\{t_{1}=\pi^{*}\left(Z_{0} / Z_{N}\right), \ldots, t_{N}=\pi^{*}\left(Z_{N-1} / Z_{N}\right)\right\}$ forms a local coordinate system of $U_{w, \mathfrak{p}}$.

Since $\left\{z_{1}, \ldots, z_{N}\right\}$ forms a set of transcendental basis of $K$ and $x_{0}, \ldots, x_{n}$ are linearly independent over $k K^{p^{m}}$, by Lemma 1 there exists a sequence of minimal integer vectors $\epsilon^{0}, \epsilon^{1}, \ldots, \epsilon^{n}$ with $0=\left|\epsilon^{0}\right|<\left|\epsilon^{1}\right| \leq \cdots \leq\left|\epsilon^{n}\right|$ such that

$$
\operatorname{det}\left(D_{\boldsymbol{z}}^{\epsilon^{i}} x_{j}\right) \neq 0
$$

To ease the notation, we will set $l_{i}:=L_{i}\left(x_{0}, \ldots, x_{n}\right)$ for $1 \leq i \leq q$. Rearranging the indices if necessary, we may assume that $\operatorname{ord}_{\mathfrak{p}}\left(l_{1}\right) \geq \operatorname{ord}_{\mathfrak{p}}\left(l_{2}\right) \geq \cdots \geq \operatorname{ord}_{\mathfrak{p}}\left(l_{q}\right)$. Then we have $\operatorname{ord}_{\mathfrak{p}}\left(l_{n+1}\right)=\cdots=\operatorname{ord}_{\mathfrak{p}}\left(l_{q}\right)=\min \left\{\operatorname{ord}_{\mathfrak{p}}\left(x_{i}\right)\right\}$ ([Wa1], Proposition 4.2). Set

$$
G=\frac{l_{1} \ldots l_{q}}{\operatorname{det}\left(D_{z}^{\epsilon^{i}} x_{j}\right)}
$$

By Proposition 3.1(a)

$$
\operatorname{det}\left(D_{\boldsymbol{z}}^{\epsilon^{i}} l_{\mu}\right)_{1 \leq \mu \leq n+1}=\operatorname{det}\left(a_{\mu \nu}\right) \operatorname{det}\left(D_{\boldsymbol{z}}^{\epsilon^{i}} x_{\nu}\right)
$$

where $l_{\mu}=\sum_{\nu=0}^{n} a_{\mu \nu} x_{\nu}$ for $1 \leq \mu \leq n+1$ and the following identities are clear:

$$
l_{n+2} \ldots l_{q}=c G \frac{\operatorname{det}\left(D_{z}^{\epsilon^{i}} l_{\mu}\right)}{l_{1} \ldots l_{n+1}}=c G \operatorname{det}\left(\frac{D_{z}^{\epsilon^{i}} l_{\mu}}{l_{\mu}}\right)
$$

where $c$ is a non-zero constant in $k$. To estimate the vanishing order of the function $\operatorname{det}\left(\frac{D_{z}^{\epsilon^{i}} l_{\mu}}{l_{\mu}}\right)_{1 \leq \mu \leq n+1}$ at $\mathfrak{p}$, we proceed as follows.

First, we express the determinant of the Wronskian $\left(D_{\boldsymbol{z}}^{\epsilon^{i}} l_{\mu}\right)$ in terms of the local coordinate system $\boldsymbol{t}$ in the neighborhood $U_{w, \mathfrak{p}}$ of $w$. By Proposition 3.1(c), we have

$$
\operatorname{det}\left(D_{\boldsymbol{z}}^{\epsilon^{i}} l_{\mu}\right)=\operatorname{det}\left(\sum_{|\boldsymbol{\alpha}|=\left|\epsilon^{i}\right|}\left(\prod_{\epsilon^{i}}^{\alpha} \frac{\partial \boldsymbol{t}}{\partial \boldsymbol{z}}\right) D_{\boldsymbol{t}}^{\boldsymbol{\alpha}} l_{\mu}\right)
$$

Put $\xi_{\mathfrak{p}}=-\min _{0 \leq i \leq n}\left\{\operatorname{ord}_{\mathfrak{p}}\left(x_{i}\right)\right\}$ and let $x_{i}^{\mathfrak{p}}=x_{i} g_{\mathfrak{p}}^{\xi_{\mathfrak{p}}}$. By Proposition 3.1(b),

$$
\operatorname{ord}_{\mathfrak{p}}\left(\operatorname{det}\left(\frac{D_{\boldsymbol{z}}^{\epsilon^{i}} l_{\mu}}{l_{\mu}}\right)\right)=\operatorname{ord}_{\mathfrak{p}}\left(\operatorname{det}\left(\frac{D_{\boldsymbol{z}}^{\epsilon^{i}} L_{\mu}\left(x_{0}^{\mathfrak{p}}, \ldots, x_{n}^{\mathfrak{p}}\right)}{L_{\mu}\left(x_{0}^{\mathfrak{p}}, \ldots, x_{n}^{\mathfrak{p}}\right)}\right)\right) .
$$


Let $m_{\mathfrak{p}}=-\min _{1 \leq i, j \leq N}\left\{\operatorname{ord}_{\mathfrak{p}}\left(\frac{\partial t_{i}}{\partial z_{j}}\right)\right\}$. Then

$$
\begin{aligned}
& \operatorname{det}\left(\frac{D_{\boldsymbol{z}}^{\epsilon^{i}} l_{\mu}}{l_{\mu}}\right) \\
& =g_{\mathfrak{p}}^{-m_{\mathfrak{p}} \sum_{i=0}^{n}\left|\epsilon^{i}\right|} \operatorname{det}\left(g_{\mathfrak{p}}^{m_{\mathfrak{p}}\left|\epsilon^{i}\right|} \frac{D_{\boldsymbol{z}}^{\epsilon^{i}} L_{\mu}\left(x_{0}^{\mathfrak{p}}, \ldots, x_{n}^{\mathfrak{p}}\right)}{L_{\mu}\left(x_{0}^{\mathfrak{p}}, \ldots, x_{n}^{\mathfrak{p}}\right)}\right) \\
& =g_{\mathfrak{p}}^{-m_{\mathfrak{p}} \sum_{i=0}^{n}\left|\epsilon^{i}\right|} \operatorname{det}\left(\sum_{|\boldsymbol{\alpha}|=\left|\epsilon^{i}\right|}\left(\prod_{\epsilon^{i}}^{\alpha} \frac{\partial \boldsymbol{t}}{\partial \boldsymbol{z}}\right) g_{\mathfrak{p}}^{m_{\mathfrak{p}}\left|\epsilon^{i}\right|} \frac{D_{\boldsymbol{t}}^{\boldsymbol{\alpha}} L_{\mu}\left(x_{0}^{\mathfrak{p}}, \ldots, x_{n}^{\mathfrak{p}}\right)}{L_{\mu}\left(x_{0}^{\mathfrak{p}}, \ldots, x_{n}^{\mathfrak{p}}\right)}\right) .
\end{aligned}
$$

By construction, $x_{i}^{\mathfrak{p}}$ and $\left(\prod_{\epsilon^{i}}^{\alpha} \frac{\partial \boldsymbol{t}}{\partial \boldsymbol{z}}\right) g_{\mathfrak{p}}^{m_{\mathfrak{p}}\left|\epsilon^{i}\right|}$ have no poles at $\mathfrak{p}$. Also by Proposition 3.2 , for $|\boldsymbol{\alpha}|=\left|\boldsymbol{\epsilon}^{i}\right|$ we have

$$
\operatorname{ord}_{\mathfrak{p}} \frac{D_{t}^{\boldsymbol{\alpha}} L_{\mu}\left(x_{0}^{\mathfrak{p}}, \ldots, x_{n}^{\mathfrak{p}}\right)}{L_{\mu}\left(x_{0}^{\mathfrak{p}}, \ldots, x_{n}^{\mathfrak{p}}\right)} \geq-\min \left\{\left|\boldsymbol{\epsilon}^{i}\right|, \operatorname{ord}_{\mathfrak{p}}\left(l_{\mu}\right)+\xi_{\mathfrak{p}}\right\} \geq-\left|\boldsymbol{\epsilon}^{i}\right| .
$$

Using the last inequality of (5.4) and the fact that $\left(\prod_{\epsilon^{i}}^{\alpha} \frac{\partial \boldsymbol{t}}{\partial \boldsymbol{z}}\right) g_{\mathfrak{p}}^{m_{\mathfrak{p}}\left|\epsilon^{i}\right|}$ does not have pole along $\mathfrak{p}$, we obtain the following:

$$
-\operatorname{ord}_{\mathfrak{p}}\left(\operatorname{det}\left(\frac{D_{\boldsymbol{z}}^{\epsilon^{i}} l_{\mu}}{l_{\mu}}\right)\right) \leq\left(m_{\mathfrak{p}}+1\right) \sum_{i=0}^{n}\left|\epsilon^{i}\right| .
$$

On the other hand, $\left|\epsilon^{i}\right| \leq\left|\epsilon^{n}\right|$ for $0 \leq i \leq n$. Then the first inequality of (5.4) gives

$$
-\operatorname{ord}_{\mathfrak{p}}\left(\operatorname{det}\left(\frac{D_{\boldsymbol{z}}^{\epsilon^{i}} l_{\mu}}{l_{\mu}}\right)\right) \leq m_{\mathfrak{p}} \sum_{i=0}^{n}\left|\boldsymbol{\epsilon}^{i}\right|+\sum_{j=1}^{n+1} \min \left\{\left|\boldsymbol{\epsilon}^{n}\right|, \operatorname{ord}_{\mathfrak{p}}\left(l_{j}\right)+\xi_{\mathfrak{p}}\right\} .
$$

Now (5.2) and (5.6) imply that

$$
\begin{aligned}
& -(q-n-1) \min _{0 \leq j \leq n}\left\{\operatorname{ord}_{\mathfrak{p}}\left(x_{j}\right)\right\} \operatorname{deg} \mathfrak{p} \\
& \leq\left(-\operatorname{ord}_{\mathfrak{p}}(G)+m_{\mathfrak{p}} \sum_{i=0}^{n}\left|\boldsymbol{\epsilon}^{i}\right|+\sum_{i=1}^{q} \min \left\{\left|\boldsymbol{\epsilon}^{n}\right|, \quad \operatorname{ord}_{\mathfrak{p}}\left(l_{i}\right)+\xi_{\mathfrak{p}}\right\}\right) \operatorname{deg} \mathfrak{p} .
\end{aligned}
$$

Similarly, (5.2) and (5.5) imply that

$$
-(q-n-1) \min _{0 \leq j \leq n}\left\{\operatorname{ord}_{\mathfrak{p}}\left(x_{j}\right)\right\} \operatorname{deg} \mathfrak{p} \leq\left(-\operatorname{ord}_{\mathfrak{p}}(G)+\left(m_{\mathfrak{p}}+1\right) \sum_{i=0}^{n}\left|\boldsymbol{\epsilon}^{i}\right|\right) \operatorname{deg} \mathfrak{p} .
$$

Summing (5.7) over all irreducible divisors $\mathfrak{p}$ which are not in $S$ and summing (5.8) over all $\mathfrak{p}$ which are in $S$, by the sum formula (4.1),

$$
\begin{aligned}
(q-n-1) h\left(x_{0}, \ldots, x_{n}\right) & \leq \sum_{\mathfrak{p} \notin S} \sum_{i=1}^{q} \min \left\{\left|\boldsymbol{\epsilon}^{n}\right|, \operatorname{ord}_{\mathfrak{p}}\left(l_{i}\right)+\xi_{\mathfrak{p}}\right\} \operatorname{deg} \mathfrak{p} \\
& +\sum_{i=0}^{n}\left|\boldsymbol{\epsilon}_{z}^{i}\right|\left(\sum_{\mathfrak{p} \in M_{K}} m_{\mathfrak{p}} \operatorname{deg} \mathfrak{p}+\sum_{\mathfrak{p} \in S} \operatorname{deg} \mathfrak{p}\right) .
\end{aligned}
$$

We will now calculate $m_{\mathfrak{p}}$ in the three cases. In case (i), we have chosen a local coordinate system $\left(t_{1}, \ldots, t_{N}\right)$ of $w$ in $U_{w, \mathfrak{p}} \cap \mathfrak{p}$ such that $\left(a t_{1}^{e_{\mathfrak{p}}}, \ldots, t_{N}\right)=$ 
$\left(u_{1}, \ldots, u_{N}\right)$ forms a local coordinate system in a neighborhood of $\pi(w)$. Note that $-\min _{1 \leq i, j \leq N}\left\{\operatorname{ord}_{\mathfrak{p}}\left(\frac{\partial u_{i}}{\partial z_{j}}\right)\right\}=0$. Therefore,

$$
\begin{aligned}
-\min _{1 \leq i, j \leq N}\left\{\operatorname{ord}_{\mathfrak{p}}\left(\frac{\partial t_{i}}{\partial z_{j}}\right)\right\} & \leq-\min _{1 \leq i, j \leq N}\left\{\operatorname{ord}_{\mathfrak{p}}\left(\frac{\partial t_{i}}{\partial u_{j}}\right)\right\}-\min _{1 \leq i, j \leq N}\left\{\operatorname{ord}_{\mathfrak{p}}\left(\frac{\partial u_{i}}{\partial z_{j}}\right)\right\} \\
& \leq e_{\mathfrak{p}}-1 .
\end{aligned}
$$

In case (ii), $t_{i}=z_{i}$. Therefore $m_{\mathfrak{p}}=0$ in this case. In case (iii), $t_{1}=z_{N}^{-1}$ and $t_{i}=z_{i-1} z_{N}^{-1}$ if $i \neq 1$. It is easy to see that $m_{\mathfrak{p}}=-2$ if $N=1$ and $m_{\mathfrak{p}}=-1$ if $N>1$. Since $\mathfrak{p}$ is unbranched in this case, the number of irreducible divisors in $\pi^{-1}\left(\left\{Z_{0}=0\right\}\right)$ equals $\operatorname{deg} V$. Therefore

$$
\sum_{\mathfrak{p}} m_{\mathfrak{p}} \operatorname{deg} \mathfrak{p} \leq \begin{cases}\operatorname{deg} \mathcal{R}_{\pi}-2 \operatorname{deg} V & \text { if } N=1, \\ \operatorname{deg} \mathcal{R}_{\pi}-\operatorname{deg} V & \text { if } N>1 .\end{cases}
$$

Equation (4.2) gives that $\mathcal{R}_{\pi}=\mathcal{K}_{V}-\pi^{*} \mathcal{K}_{\mathbb{P}^{N}}=\mathcal{K}_{V}+(N+1) \pi^{*}[H]$, where $H$ is a generic hyperplane of $\mathbb{P}^{N}$. Therefore

$$
\sum_{\mathfrak{p}} m_{\mathfrak{p}} \operatorname{deg} \mathfrak{p} \leq \begin{cases}\operatorname{deg} \mathcal{K}_{V} & \text { if } N=1, \\ \operatorname{deg} \mathcal{K}_{V}+N \operatorname{deg} V & \text { if } N>1 .\end{cases}
$$

If $K$ has positive characteristic, then Lemma 2 implies

$$
\sum_{i=0}^{n}\left|\epsilon^{i}\right| \leq \sum_{i=0}^{n} i p^{m-1}=\frac{n(n+1)}{2} p^{m-1}
$$

If $p=0$, then by Remark 2 of Lemma 2, we have

$$
\sum_{i=0}^{n}\left|\epsilon^{i}\right| \leq \sum_{i=0}^{n} i=\frac{n(n+1)}{2}
$$

Now the proof of Theorem 1 is completed.

Proof of the Corollary. Let $V=\mathbb{P}^{N}$ in Theorem 1. Let $U_{i}=\left\{\left[Z_{0}, \ldots, Z_{N}\right] \mid Z_{i} \neq\right.$ $0\}, i=0, \ldots, N$, be the standard open coverings of $V$. Choose $z_{1}=Z_{1} / Z_{0}, \ldots, z_{N}=$ $Z_{N} / Z_{0}$ to be the fixed transcendental basis which is also the coordinate system in $U_{0}$. The polynomial ring $k\left[z_{1}, \ldots, z_{n}\right]$ are rational functions on $\mathbb{P}^{N}$ which are regular on $U_{0}$. Denote by $\mathfrak{p}_{\infty}$ the divisor defined by $Z_{0}=0$. Let $\mathfrak{p} \neq \mathfrak{p}_{\infty}$ be a prime divisor of $\mathbb{P}^{N}$; then $\mathfrak{p}$ corresponds an irreducible polynomial of $k\left[z_{1}, \ldots, z_{N}\right]$. Since $k\left[z_{1}, \ldots, z_{N}\right]$ is a unique factorization domain and $f_{0}, \ldots, f_{n}$ are relatively prime, we have $\min _{0 \leq j \leq n}\left\{\operatorname{ord}_{\mathfrak{p}} f_{j}\right\}=0$ and the given set of elements $f_{0}, \ldots, f_{n}$ has height $h\left(\left[f_{0}, \ldots, f_{n}\right]\right)=\max \left\{\operatorname{deg} f_{0}, \ldots, \operatorname{deg} f_{n}\right\}$.

Let $K=k\left(z_{1}, \ldots, z_{N}\right)$ be the field of rational functions in $N$-variables. Let $S$ be the subset of $M_{K}$ consisting of $\mathfrak{p}_{\infty}$ only. Note that $\operatorname{deg} \mathcal{K}_{\mathbb{P}^{N}}=-(N+1)$. It is clear the corollary follows from Theorem 1.

\section{A REFinement on the ORDERS OF the ITERATED DERIVATIVES}

We assume that $p$ is positive in this section. Although the bounds of the order of the minimal integer vectors $\boldsymbol{\epsilon}^{0}, \ldots, \boldsymbol{\epsilon}^{n}$ given in Lemma 1 and Lemma 2 are the best possible in some special cases, they may not be sharp enough in most cases. In the following theorem we provide a better bound for the order of the integer vectors $\epsilon^{0}, \ldots, \epsilon^{n}$ in terms of the dimension of the vector spaces spanned by $x_{0}, \ldots, x_{n}$ over 
$K^{p^{\gamma}}$ for $0 \leq \gamma \leq m$. Assume that $p^{c N} \leq n<p^{(c+1) N}$. Since the field extension degree $\left[K: K^{p^{c}}\right]=p^{c N}$ by Proposition 2.3 , it is necessary that $m \geq c+1$ if $x_{0}, \ldots, x_{n}$ are linearly independent over $K^{p^{m}}$. Denote by $l_{\gamma}+1$ the dimension of the vector space spanned by $x_{0}, \ldots, x_{n}$ over $K^{p^{\gamma}}$ for $0 \leq \gamma \leq m$. Let $0=\gamma(0)<$ $\gamma(1)<\cdots<\gamma(u) \leq m$ be a sequence of integers between 0 and $m$ such that $0=l_{\gamma(0)}<l_{\gamma(1)}<\cdots<l_{\gamma(u)}=n$ and $l_{\gamma(\delta)}=l_{\gamma(\delta)+1}=\cdots=l_{\gamma(\delta+1)-1}$, for $0 \leq \delta<u$. With the above notation, we have the following

Theorem 2. Let $x_{0}, \ldots, x_{n} \in K$. Then $x_{0}, \ldots, x_{n}$ are linearly independent over $K_{m}$ if and only if there exist integer vectors $\epsilon^{i}$ with $0=\left|\epsilon^{0}\right|<\left|\epsilon^{1}\right| \leq \cdots \leq\left|\epsilon^{n}\right|$ such that

$$
\operatorname{det}\left(D_{z}^{\epsilon^{i}} x_{j}\right)_{0 \leq i, j \leq n} \neq 0
$$

and the integer vectors $\epsilon^{i}=\left(\epsilon_{1}^{i}, \ldots, \epsilon_{N}^{i}\right), i=0,1, \ldots, n$, satisfy the following conditions for $1 \leq s \leq l_{\gamma(\delta)}-l_{\gamma(\delta-1)}$ where $1 \leq \delta \leq u$ as defined above:

(A) $\epsilon_{j}^{l_{\gamma(\delta-1)}+s} \leq \min \left\{s p^{\gamma(\delta)-1}, p^{\gamma(\delta)}-1\right\}, \quad 1 \leq j \leq N$

(B) $\left|\boldsymbol{\epsilon}^{l_{\gamma(\delta-1)}+s}\right| \leq\left(l_{\gamma(\delta-1)}+s\right) p^{\gamma(\delta)-1}$

(C) there exists a component $\epsilon_{j}^{l_{\gamma(\delta-1)}+s}$ in the corresponding vector $\boldsymbol{\epsilon}^{l_{\gamma(\delta-1)}+s}$ such that $\epsilon_{j}^{l_{\gamma(\delta-1)}+s} \geq p^{\gamma(\delta)-1}$.

In particular, there exists a component $\epsilon_{j}^{l_{\gamma(\delta-1)}+1}$ in the corresponding vector $\boldsymbol{\epsilon}^{l_{\gamma(\delta-1)}+1}$ such that $\epsilon_{j}^{l_{\gamma(\delta-1)}+1}=p^{\gamma(\delta)-1}$.

We recall the following result which is needed for the proof of Theorem 2.

Proposition 6.1. Let $p$ be a prime number. Let $a=\sum_{i \geq 0} a_{i} p^{i}$ and $b=\sum_{i \geq 0} b_{i} p^{i}$, with $0 \leq a_{i}, b_{i} \leq p-1$, be the $p$-adic expansions of the nature numbers $a$ and $b$. Then $\left(\begin{array}{l}a \\ b\end{array}\right)$ is not divisible by $p$ if and only if $a_{i} \geq b_{i}$ for all $i \geq 0$.

Proof. See [HS], or [GV].

Proof of Theorem 2. The "if" part of the theorem comes from Lemma 1. Therefore we only need to show the "only if" part.

Without loss of generality we may assume that $x_{0}, \ldots, x_{l_{\gamma(\delta)}}$ are linearly independent over $K^{p^{\gamma(\delta)}}$. By the definition of $\gamma(\delta), x_{0}, \ldots, x_{l_{\gamma(\delta-1)}+s}$ are linearly dependent over $K^{p^{\gamma(\delta)-1}}$ for $1 \leq s \leq l_{\gamma(\delta)}-l_{\gamma(\delta-1)}$. Then the row vectors

$$
\left(D_{\boldsymbol{z}}^{\boldsymbol{\alpha}} x_{0}, D_{\boldsymbol{z}}^{\boldsymbol{\alpha}} x_{1}, \ldots, D_{\boldsymbol{z}}^{\boldsymbol{\alpha}} x_{l_{\gamma(\delta)}}\right) \text {, for all }|\boldsymbol{\alpha}| \text { such that } \alpha_{j}<p^{\gamma(\delta)-1} \text { for all } j
$$

are linearly dependent. Hence, if $\boldsymbol{\epsilon}^{0}, \ldots, \boldsymbol{\epsilon}^{n}$ satisfy $(*)$, then they must satisfy (C).

We now claim that there exists a sequence of integer vectors which satisfies (A) and (B) such that $(*)$ holds. This will be done by induction on $n$. When $n=0$, this is trivial. The proof will then be completed by two induction steps. First we show that if the claim is true for $n=l_{\gamma(\delta-1)}$, then it is true for $n=l_{\gamma(\delta-1)}+1$. We then show that if the claim is true for $n=l_{\gamma(\delta-1)}+s$, then it is true for $n=l_{\gamma(\delta-1)}+s+1$, where $1 \leq s<l_{\gamma(\delta)}-l_{\gamma(\delta-1)}$.

Now we start the first part of the induction steps. By induction, we assume that the claim is true for $n=l_{\gamma(\delta-1)}$. Let $x_{0}, \ldots, x_{l_{\gamma(\delta-1)}+1}$ be linearly independent over $K^{p^{\gamma(\delta)}}$ and assume the conclusion of the claim does not hold. Then the following 
vectors, $0 \leq i \leq l_{\gamma(\delta-1)}+1$,

$\underline{\boldsymbol{x}}_{i}=\left(x_{i}, \ldots D_{\boldsymbol{z}}^{\boldsymbol{\alpha}} x_{i}, \ldots\right)$ with $\boldsymbol{\alpha} \leq\left(p^{\gamma(\delta)-1}, \ldots, p^{\gamma(\delta)-1}\right)$ and $|\boldsymbol{\alpha}| \leq\left(l_{\gamma(\delta-1)}+1\right) p^{\gamma(\delta)-1}$

are linearly dependent over $K$. Then there exist $a_{0}, \ldots, a_{l_{\gamma(\delta-1)}+1} \in K$, not all zeros, such that

$(6.1)$

$\sum_{i=0}^{l_{\gamma(\delta-1)}+1} a_{i} D_{\boldsymbol{z}}^{\boldsymbol{\alpha}} x_{i}=0 \quad$ for $\boldsymbol{\alpha} \leq\left(p^{\gamma(\delta)-1}, \ldots, p^{\gamma(\delta)-1}\right)$ and $|\boldsymbol{\alpha}| \leq\left(l_{\gamma(\delta-1)}+1\right) p^{\gamma(\delta)-1}$.

Since $x_{0}, \ldots, x_{l_{\gamma(\delta-1)}}$ are linearly independent over $K^{\gamma^{\gamma(\delta-1)}}$, by the induction assumption that the vectors $\underline{\boldsymbol{x}}_{0}, \ldots, \underline{\boldsymbol{x}}_{l_{\gamma(\delta-1)}}$ are linearly independent over $K$. Hence $a_{l_{\gamma(\delta-1)}+1} \neq 0$ and without loss of generality we can assume that $a_{l_{\gamma(\delta-1)}+1}=1$. We shall show that $a_{i} \in K^{p^{\gamma(\delta)}}$ and then $(6.1)$ with $\boldsymbol{\alpha}=(0, \ldots, 0)$ will give a contradiction that completes this step of proof. By Proposition 2.2(a), it suffices to show that $D_{z_{j}}^{\left(p^{r}\right)} a_{i}=0$ for $0 \leq r \leq \gamma(\delta)-1$ and $1 \leq j \leq N$. This will be done by induction. For $r=0$, applying $D_{z_{j}}$ to (6.1) we have

$$
\left(\alpha_{j}+1\right) \sum_{i=0}^{l_{\gamma(\delta-1)}+1} a_{i} D_{z}^{\left(\alpha_{1}, \ldots, \alpha_{j}+1, \ldots, \alpha_{N}\right)} x_{i}+\sum_{i=0}^{l_{\gamma(\delta-1)}} D_{z_{j}} a_{i} D_{z}^{\alpha} x_{i}=0 .
$$

Then from (6.1), we have $\sum_{i=0}^{l_{\gamma(\delta-1)}} D_{z_{j}} a_{i} D_{z}^{\boldsymbol{\alpha}} x_{i}=0$, for $\alpha_{j} \leq p^{\gamma(\delta)-1}-1, \alpha_{q} \leq$ $p^{\gamma(\delta)-1}$ if $q \neq j$ and $|\boldsymbol{\alpha}| \leq\left(l_{\gamma(\delta-1)}+1\right) p^{\gamma(\delta)-1}-1$. Deduce from the induction assumption that the following vectors for $0 \leq i \leq l_{\gamma(\delta-1)}$

$$
\left(x_{i}, \ldots, D_{\boldsymbol{z}}^{\boldsymbol{\alpha}} x_{i}, \ldots\right) \text { with } \boldsymbol{\alpha} \leq\left(p^{\gamma(\delta-1)}, \ldots, p^{\gamma(\delta-1)}\right) \text { and }|\boldsymbol{\alpha}| \leq l_{\gamma(\delta-1)} p^{\gamma(\delta-1)-1}
$$

are linearly independent over $K$. Hence $D_{z_{j}} a_{i}=0$ for $0 \leq i \leq l_{\gamma(\delta-1)}$.

Assume that $D_{z_{j}}^{\left(p^{\nu}\right)} a_{i}=0$ for $1 \leq j \leq N, 0 \leq \nu \leq r-1$ and $0 \leq i \leq l_{\gamma(\delta-1)}$. Then $a_{i} \in K^{p^{r}}$ for $0 \leq i \leq l_{\gamma(\delta-1)}$. Therefore

$$
D_{\boldsymbol{z}}^{\boldsymbol{\beta}} a_{i}=0 \quad \text { for }(0, \ldots, 0) \neq \boldsymbol{\beta} \leq\left(p^{r}-1, \ldots, p^{r}-1\right) \text { and } 0 \leq i \leq l_{\gamma(\delta-1)} .
$$

Let the operator $D_{z_{j}}^{\beta_{j}}$ with $\beta_{j} \leq p^{r}-1$ apply to (6.1) for $\boldsymbol{\alpha}$ with $\alpha_{j}=p^{\gamma(\delta)-1}$. Then by Proposition 2.1 and (6.4) we have

$$
\left(\begin{array}{c}
p^{\gamma(\delta)-1}+\beta_{j} \\
\beta_{j}
\end{array}\right) \sum_{i=0}^{l_{\gamma(\delta-1)}+1} a_{i} D_{\boldsymbol{z}}^{\left(\alpha_{1}, \ldots, p^{\gamma(\delta)-1}+\beta_{j}, \ldots, \alpha_{N}\right)} x_{i}=0
$$

Since $\beta_{j}<p^{\gamma(\delta)-1}$, from Proposition 6.1 we have $\left(\begin{array}{c}p^{\gamma(\delta)-1}+\beta_{j} \\ \beta_{j}\end{array}\right) \neq 0(\bmod p)$. Therefore,

$$
\sum_{i=0}^{l_{\gamma(\delta-1)}+1} a_{i} D_{\boldsymbol{z}}^{\left(\alpha_{1}, \ldots, \alpha_{j}+\beta_{j}, \ldots, \alpha_{N}\right)} x_{i}=0, \quad \text { for } \boldsymbol{\alpha} \text { in }(6.1) \text { and } \beta_{j} \leq p^{r}-1 .
$$

Now apply the operator $D_{z_{j}}^{p^{r}}$ to (6.1); then as a result of (6.6),

$$
\sum_{i=0}^{l_{\gamma(\delta-1)}} D_{z_{j}}^{p^{r}} a_{i} D_{\boldsymbol{z}}^{\boldsymbol{\alpha}} x_{i}=0, \text { for } \boldsymbol{\alpha} \leq\left(p^{\gamma(\delta)-1}-1, \ldots, p^{\gamma(\delta)-1}-1\right) \text { and }|\boldsymbol{\alpha}| \leq l_{\gamma(\delta-1)} p^{\gamma(\delta)-1} \text {. }
$$


Again since the vectors in (6.3) are linearly independent over $K$, it follows that $D_{z_{j}}^{p^{r}} a_{i}=0$ for $0 \leq i \leq l_{\gamma(\delta-1)}$. This completes the first step of the induction proof of the claim.

It remains to show that if the claim is true for $n=l_{\gamma(\delta)-1}+s$, then it is true for $n=l_{\gamma(\delta)-1}+s+1,1 \leq s<l_{\gamma(\delta)}-l_{\gamma(\delta-1)}$. If $(s+1) p^{\gamma(\delta)-1} \geq p^{\gamma(\delta)}-1$, then this induction step is similar to the proof of Lemma 1. If $(s+1) p^{\gamma(\delta)-1}<p^{\gamma(\delta)}-1$, this induction step can be showed by Lemma 1 and Lemma 2. We will omit these arguments.

\section{ACKNOWLEDGMENTS}

The authors thank P.-M. Wong for many helpful discussions. We also thank the referee for useful comments on the presentation of this paper and pointing out a mistake in an early version.

\section{REFERENCES}

[BM] Brownawell, D and Masser, D., Vanishing sums in function fields, Math. Proc. Cambridge Philos. Soc. 100 (1986), 427-434. MR 87k:11080

[BPV] Barth, W., Peters, C. and Van de Ven, A., Compact complex surfaces, Springer-Verlag, 1984. MR 86c:32026

[Bu] Buium, A., The abc theorem for abelian varieties, International Math. Research Notices 5 (1994), 219-233. MR 95c:11074

[Fu] Fujimoto, H., Non-integrated defect relation for meromorphic maps of complete Kähler manifolds into $\mathbb{P}_{1}^{N}(\mathbb{C}) \times \cdots \times \mathbb{P}_{k}^{N}(\mathbb{C})$, Japan. J. Math. 11 (1985), 233-264. MR 88m:32049

[GV] Garcia, A. and Voloch, J. F., Wronskians and linear independence in fields of prime characteristic, Manuscripta Math. 59 (1987), 457-469. MR 88m:12005

[HS] Hasse, H. and Schmidt, F. K., Noch eine Bergründung der Theorie der höheren Differentialquotienten in einem algebraischen Funktionenkörper einer Unbestimmtten, J. Reine Angew. Math. 177 (1937), 215-237.

[La1] Lang, S., Algebra, Addison-Wesley, 1984. MR 86j:00003

[La2] Lang, S., Fundamentals of Diophantine Geometry, Springer-Verlag, 1983. MR 85j:11005

[Ma] Mason, R. C., Diophantine equations over function fields LMS. Lecture Notes 96, Cambridge Univ. Press, 1984. MR 86b:11026

[Mum] Mumford, D., Algebraic geometry I. Complex Projective Varieties, Springer-Verlag, 1976. MR 56:11992

[No] Noguchi, J., Nevanlinna-Cartan theory and a Diophantine equation over function fields, J. Reine Angew. Math. 487 (1997), 61-83. MR 98d:11076

[Ok] Okugawa, K., Basic properties of differential fields of an arbitrary characteristic and the Picard-Vessiot theory, J. Math. Kyoto Univ. 2 (1963), 295-322. MR 27:5754

[Se] Serre, J.-P., Lectures on the Mordell-Weil theorem, Vieweg, 1989. MR 90e:11086

[Si] Silverman, J. H., The S-unit equation over function fields, Proc. Camb. Philos. Soc. 95 (1984), 3-4. MR 85e:11018

[SS] Shapiro, H. N. and Sparer, G. H., Extension of a theorem of Mason, Comm. Pure. Appl. Math. XLVII (1994), 711-718. MR 95c:11036

[SV] Stöhr, K-O. and Voloch, J. F., Weierstrass points and curve over function fields, Proc. London Math. Soc. (3) 52 (1986), 1-19. MR 87b:14010

[SW] Stoll, W. and Wong, P.-M., Second main theorem of Nevanlinna theory for nonequidimensional meromorphic maps, Amer. J. of Math. 116 (1994), 1031-1071. MR 95g:32042

[Voj] Vojta, P., Diophantine approximation and value distribution theory, Lecture Notes in Math., vol. 1239, Springer, 1987. MR 91k:11049

[Vol] Voloch, J. F., Diagonal equations over function fields, Bol. Soc. Brazil Math. 16 (1985), 29-39. MR 87g:11157

[Wa1] Wang, J., T.-Y., The truncated second main theorem of function fields, J. of Number Theory 58 (1996), 139-157. MR 97c:11074 
[Wa2] Wang, J., T.-Y., A note on Wronskians and the ABC theorem, Manuscripta Math. 98 (1999), 255-264. MR 2000d:11086

[Wa3] Wang, J., T.-Y., ABC estimate, integral points, and geometry of $P^{n}$ minus hyperplanes, Mathematical Research Letters 6 (1999), 357-370. MR 2000j:11114

[Ye] Ye, Z., On Nevanlinna's second main theorem in projective spaces, Invent. Math 122 (1995), 475-507. MR 96j:32030

Department of Mathematics, National Central University, Taiwan

E-mail address: hsia@math.ncu.edu.tw

Institute of Mathematics, Academia Sinica, Nankang 115, Taipei, Taiwan

E-mail address: jwang@math.sinica.edu.tw 